\title{
Organic transformations catalyzed by palladium nanoparticles on carbon nanomaterials
}

\author{
BHAIRI LAKSHMINARAYANA, LODI MAHENDAR, JHONTI CHAKRABORTY, \\ GEDU SATYANARAYANA* and CH SUBRAHMANYAM* \\ Department of Chemistry, Indian Institute of Technology Hyderabad, Kandi, Medak District, Sangareddy, \\ Telangana 502 285, India \\ E-mail: gvsatya@iith.ac.in; csubbu@iith.ac.in
}

MS received 8 August 2017; revised 16 March 2018; accepted 16 March 2018; published online 20 April 2018

\begin{abstract}
An efficient $\mathrm{C}-\mathrm{C}$ bond coupling reactions (Suzuki-Miyaura and Glaser) catalyzed by PdO/GO nano-catalyst is presented. In addition, PdO/MWCNT nano-catalyst-mediated domino one-pot synthesis of 2-alkyl/2-aryl benzofurans has been accomplished from 2-iodophenols and terminal alkynes. The formation of benzofurans proceeds through intermolecular Sonogashira reaction followed by intramolecular nucleophilic addition of internal hydroxyl group onto the acetylenic bond. The catalyst $\mathrm{PdO} / \mathrm{GO}$ has been reused successfully, with nearly no loss of activity up to 5 cycles.
\end{abstract}

Keywords. Graphene oxide (GO); multi-walled carbon nanotubes (MWCNT); C-C coupling reaction; benzofuran derivatives.

\section{Introduction}

From a green chemistry point of view, heterogeneous catalysis holds paramount advantages over its homogeneous counterparts such as, easy separation, reusability and stability of the catalyst. However, dispersion of the chosen catalytic material on a suitable support is still a challenge to the scientific community. This problem is even more complex in nanomaterial catalysts, due to aggregation problems. In this context, hybrid carbon materials such as graphene oxide (GO), reduced graphene oxide (RGO), multi-walled carbon nanotubes (MWCNT), single-walled carbon nanotubes (SWCNT), and carbon nanofiber (CNF) have been tested as supports. ${ }^{1-4}$ Palladium is one of the most widely used metals for organic transformations. However, unsupported $[\mathrm{Pd}]$-nanoparticles agglomerate or sinter upon heating. ${ }^{5-8}$ Carbon-supported [Pd]-based nanoparticles proved to be highly active catalytic systems and furnished the coupling products in high yields with good selectivity. These advantageous features are attributed to the electronic, mechanical and thermal properties of the carbon support. ${ }^{9-13}$
Until now, homogenous [Pd]-catalysis has been broadly explored for organic coupling transformations. In modern organic synthesis, the development of environmentally benign organic transformations is highly desirable. ${ }^{14-17}$ Recently, we have reported the syntheses and characterization of $\mathrm{PdO}$ nanoparticles impregnated on various nano-carbon supports (SWCNT, MWCNT, CNF, GO and RGO). ${ }^{18,19}$ Also, the efficiency of these ligand-free catalysts has been demonstrated with the Heck reaction between iodoarenes and olefins. Among them, PdO/GO catalyst was found to be the best, due to a high degree of surface-bound oxygenated moieties. The combined effects of high surface area, mesoporous nature, $\pi$-interactions, optimum electron conductivity of GO favored the activity of $\mathrm{PdO} / \mathrm{GO}$ nanohybrids, for Heck cross-coupling reactions. Encouraged by these initial results, we are reporting the extension of the scope of this versatile system for other organic coupling transformations. This communication deals with the applications of ligand-free $\mathrm{PdO} / \mathrm{GO}$, for C-C bond forming Suzuki-Miyaura and Glaser coupling reactions, respectively. In addition, ligand-free heterogeneous $\mathrm{PdO} / \mathrm{MWCNT}$-catalyzed domino one-pot synthesis of

\footnotetext{
*For correspondence

Electronic supplementary material: The online version of this article (https://doi.org/10.1007/s12039-018-1449-9) contains supplementary material, which is available to authorized users.
} 
2-arylbenzofurans through intermolecular Sonogashira coupling followed intermolecular nucleophilic addition to the acetylenic bond, is also described.

\section{Experimental}

\subsection{General considerations}

IR spectra were recorded on a FTIR spectrophotometer. ${ }^{1} \mathrm{H}$ NMR spectra were recorded on $400 \mathrm{MHz}$ spectrometer at $295 \mathrm{~K}$ in $\mathrm{CDCl}_{3}$; chemical shifts $(\delta \mathrm{ppm})$ and coupling constants $(\mathrm{Hz})$ are reported in standard fashion with reference to either internal standard tetramethylsilane (TMS) $\left(\delta_{\mathrm{H}}=0.00 \mathrm{ppm}\right)$ or $\mathrm{CHCl}_{3}\left(\delta_{\mathrm{H}}=7.25 \mathrm{ppm}\right) .{ }^{13} \mathrm{C} \mathrm{NMR}$ spectra were recorded on $100 \mathrm{MHz}$ spectrometer at RT in $\mathrm{CDCl}_{3}$; chemical shifts ( $\delta \mathrm{ppm}$ ) are reported relative to $\mathrm{CHCl}_{3}$ $[\delta \mathrm{C}=77.00 \mathrm{ppm}$ (central line of triplet) $]$. In the ${ }^{13} \mathrm{C} \mathrm{NMR}$, the nature of carbons $\left(\mathrm{C}, \mathrm{CH}, \mathrm{CH}_{2}\right.$, and $\left.\mathrm{CH}_{3}\right)$ was determined by recording the DEPT-135 spectra and is given in parentheses and noted as $\mathrm{s}=$ singlet (for $\mathrm{C}$ ), $\mathrm{d}=\operatorname{doublet}($ for $\mathrm{CH}$ ), $\mathrm{t}=$ triplet (for $\mathrm{CH}_{2}$ ) and $\mathrm{q}=$ quartet (for $\mathrm{CH}_{3}$ ). In the ${ }^{1} \mathrm{H} \mathrm{NMR}$, the following abbreviations were used throughout: $\mathrm{s}=$ singlet, $\mathrm{d}=$ doublet, $\mathrm{t}=$ triplet, $\mathrm{q}=$ quartet, qui $=$ quintet, $\mathrm{m}=$ multiplet and br $\mathrm{s} .=$ broad singlet. The assignment of signals was confirmed by ${ }^{1} \mathrm{H},{ }^{13} \mathrm{C} \mathrm{CPD}$ (carbon proton decoupled), and DEPT spectra. High-resolution mass spectra (HR-MS) were recorded using Q-TOF multimode source. Melting points were determined on an electrothermal melting point apparatus and are uncorrected. Iodophenols, $\mathrm{PdO}$ on MWCNT, terminal alkynes and $\mathrm{K}_{2} \mathrm{CO}_{3}$ were commercially available (local made) used without further purification.

Reactions were monitored by TLC on silica gel using a combination of petroleum ether and ethyl acetate as eluents. Solvents were distilled prior to use; petroleum ether with a boiling range of 40 to $60{ }^{\circ} \mathrm{C}$ was used. Acme's silica gel (60-120 mesh) was used for column chromatography (approximately $20 \mathrm{~g}$ per one gram of crude material).

\subsection{General procedure 1 (for the synthesis of biphenyls 3)}

In an oven-dried Schlenk tube iodoarenes $\mathbf{1}(0.25 \mathrm{mmol})$, arylboroic acids $2(0.5 \mathrm{mmol}), \mathrm{PdO} / \mathrm{GO}$ nano catalyst $(5 \mathrm{~mol} \%)$, $\mathrm{K}_{2} \mathrm{CO}_{3}(0.5 \mathrm{mmol})$ and solvent (DMSO) $(1.0 \mathrm{~mL})$ were added. The resulting reaction mixture was stirred at $120{ }^{\circ} \mathrm{C}$ for 5-30 min. The progress of the reaction was monitored by TLC. After completion of reaction, the reaction mixture was allowed to cool to room temperature, then diluted with (approximately $10 \mathrm{~mL}$ ) ethyl acetate and $\mathrm{NH}_{4} \mathrm{Cl}$ solution (approximately $10 \mathrm{~mL}$ ) was added followed by extraction with ethyl acetate. The organic layers were dried with $\mathrm{Na}_{2} \mathrm{SO}_{4}$ and concentrated in reduced vacuum. Purification of the residue by silica gel column chromatography using distilled petroleum ether/ethyl acetate as the eluent furnished the biphenyls.

\subsection{General procedure 2 (for the synthesis of symmetrical 1,3-diynes 5)}

In an oven-dried Schlenk tube terminal alkynes $4(0.5 \mathrm{mmol})$, $\mathrm{Pd} / \mathrm{GO}$ nano catalyst $(5 \mathrm{~mol} \%), \mathrm{K}_{2} \mathrm{CO}_{3}(1 \mathrm{mmol})$ and solvent (DMSO) $(1.0 \mathrm{~mL})$ were added. The resulting reaction mixture was stirred at $120^{\circ} \mathrm{C}$ for $2 \mathrm{~h}$. The progress of the reaction was monitored by TLC. After completion of reaction, the reaction mixture was allowed to cool to room temperature, then diluted with $(\sim 10 \mathrm{~mL})$ ethyl acetate and $\mathrm{NH}_{4} \mathrm{Cl}$ solution $(\sim 10 \mathrm{~mL})$ was added fallowed by extraction with ethyl acetate. The organic layers were dried with $\mathrm{Na}_{2} \mathrm{SO}_{4}$ and concentrated in reduced vacuum. Purification of the residue by silica gel column chromatography using distilled petroleum ether/ethyl acetate as the eluent furnished the cinnamates and stilbenes.

\subsection{General procedure 3 (for the synthesis of benzofurans 7)}

In an oven-dried Schlenk tube 2-iodophenol $6(0.5 \mathrm{mmol})$, terminal alkyne 4 ( $0.5 \mathrm{mmol})$, PdO/MWCNT (5 mol\%), $\mathrm{K}_{2} \mathrm{CO}_{3}$ $(1 \mathrm{mmol})$ and solvent (DMSO) $(1.0 \mathrm{~mL})$ were added. The resulting reaction mixture was stirred at $120^{\circ} \mathrm{C}$ for $24 \mathrm{~h}$. The progress of the reaction was monitored by TLC. After completion of reaction, the reaction mixture was allowed to cool to room temperature, then diluted with $(10 \mathrm{~mL})$ ethyl acetate and $\mathrm{NH}_{4} \mathrm{Cl}(10 \mathrm{~mL})$ was added fallowed by extraction with ethyl acetate. The organic layers were dried $\left(\mathrm{Na}_{2} \mathrm{SO}_{4}\right)$ and concentrated in vacuum. Purification of the residue by silica gel column chromatography using petroleum ether/ethyl acetate as the eluent furnished the benzofuran 7 .

\subsection{Characterization data}

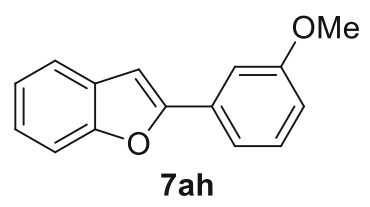

This compound was prepared according to the GP and isolated as colorless liquid $94 \%$ yield $(105 \mathrm{mg}$ ). [TLC (petroleum ether/ethyl acetate 9:1, $R_{\mathrm{f}}(\mathbf{6 a})=0.50, \mathrm{R}_{\mathrm{f}}(\mathbf{4} \mathbf{h})=0.60, \mathrm{UV}$ detection]. ${ }^{1} \mathrm{H}$ NMR $\left(\mathrm{CDCl}_{3}, 400 \mathrm{MHz}\right): \delta=7.59(\mathrm{~d}, J=$ $7.3 \mathrm{~Hz}, 1 \mathrm{H}), 7.53(\mathrm{~d}, J=7.3 \mathrm{~Hz}, 1 \mathrm{H}), 7.46(\mathrm{~d}, J=8.8 \mathrm{~Hz}$, $1 \mathrm{H}), 7.42(\mathrm{~s}, 1 \mathrm{H}), 7.36(\mathrm{dd}, J=7.8$ and $7.8 \mathrm{~Hz}, 1 \mathrm{H}), 7.29$ (dd, $J=8.3$ and $7.3 \mathrm{~Hz}, 1 \mathrm{H}), 7.23(\mathrm{~d}, J=7.3 \mathrm{~Hz}, 1 \mathrm{H}), 7.03$ (s, $1 \mathrm{H}), 6.91(\mathrm{dd}, J=7.3$ and $2.0 \mathrm{~Hz}, 1 \mathrm{H}), 3.89(\mathrm{~s}, 3 \mathrm{H}) \mathrm{ppm}$. ${ }^{13} \mathrm{C}$ NMR $\left(\mathrm{CDCl}_{3}, 100 \mathrm{MHz}\right): \delta=159.9\left(\mathrm{C}_{\mathrm{q}}\right), 155.7\left(\mathrm{C}_{\mathrm{q}}\right)$, $154.8\left(\mathrm{C}_{\mathrm{q}}\right), 131.7\left(\mathrm{C}_{\mathrm{q}}\right), 129.8(\mathrm{CH}), 129.1\left(\mathrm{C}_{\mathrm{q}}\right), 124.3(\mathrm{CH})$, $122.9(\mathrm{CH}), 120.9(\mathrm{CH}), 117.5(\mathrm{CH}), 114.4(\mathrm{CH}), 111.2(\mathrm{CH})$, $110.1(\mathrm{CH}), 101.6(\mathrm{CH}), 55.3\left(\mathrm{CH}_{3}\right) \mathrm{ppm}$. IR (MIR-ATR, $\left.4000-600 \mathrm{~cm}^{-1}\right): v_{\max }=2921,2851,1482,1460,1380$, 
1123, 1109, 1011, $928 \mathrm{~cm}^{-1}$. HR-MS $\left(\mathrm{ESI}^{+}\right) \mathrm{m} / \mathrm{z}$ calculated for $\left[\mathrm{C}_{15} \mathrm{H}_{12} \mathrm{O}_{2}\right]^{+}=[\mathrm{M}]^{+}$: 224.0832; found: 224.0840 .<smiles>COc1cccc(-c2cc3c(C=O)ccc(OC)c3o2)c1</smiles>

This compound was prepared according to the GP and isolated as pale yellow color viscous liquid $90 \%$ yield $(127 \mathrm{mg}$ ). [TLC (petroleum ether/ethyl acetate $8: 2, \mathrm{R}_{\mathrm{f}}(\mathbf{6 b})=0.50$, $\mathrm{R}_{\mathrm{f}}(4 \mathrm{~h})=0.60$, UV detection]. ${ }^{1} \mathrm{HNMR}\left(\mathrm{CDCl}_{3}, 400 \mathrm{MHz}\right)$ : $\delta=10.03(\mathrm{~s}, 1 \mathrm{H}), 7.78(\mathrm{~s}, 1 \mathrm{H}), 7.65(\mathrm{~d}, J=8.3 \mathrm{~Hz}, 1 \mathrm{H}), 7.53$ $(\mathrm{d}, J=7.8 \mathrm{~Hz}, 1 \mathrm{H}), 7.44(\mathrm{dd}, J=2.4$ and $2.0 \mathrm{~Hz}, 1 \mathrm{H}), 7.36$ (dd, $J=8.3$ and $7.8 \mathrm{~Hz}, 1 \mathrm{H}), 6.93(\mathrm{dd}, J=8.8$ and $3.2 \mathrm{~Hz}$, $1 \mathrm{H}), 6.88$ (d, $J=8.3 \mathrm{~Hz}, 1 \mathrm{H}), 4.11$ (s, 3H), 3.88 (s, 3H) ppm. ${ }^{13} \mathrm{C} \mathrm{NMR}\left(\mathrm{CDCl}_{3}, 100 \mathrm{MHz}\right): \delta=190.8\left(\mathrm{C}_{\mathrm{q}}\right), 159.9\left(\mathrm{C}_{\mathrm{q}}\right)$, $158.8\left(\mathrm{C}_{\mathrm{q}}\right), 149.8\left(\mathrm{C}_{\mathrm{q}}\right), 144.0\left(\mathrm{C}_{\mathrm{q}}\right), 132.1(\mathrm{CH}), 130.9\left(\mathrm{C}_{\mathrm{q}}\right)$, $129.9(\mathrm{CH}), 129.8\left(\mathrm{C}_{\mathrm{q}}\right), 122.9\left(\mathrm{C}_{\mathrm{q}}\right), 118.0(\mathrm{CH}), 115.5(\mathrm{CH})$, $110.3(\mathrm{CH}), 106.1(\mathrm{CH}), 102.2(\mathrm{CH}), 56.4\left(\mathrm{CH}_{3}\right), 55.4\left(\mathrm{CH}_{3}\right)$ ppm. IR (MIR-ATR, 4000-600 $\mathrm{cm}^{-1}$ ): $v_{\max }=2922,2850$, 1478, 1380, 1201, 1165, 1011, $920 \mathrm{~cm}^{-1}$. HR-MS $\left(\mathrm{ESI}^{+}\right) \mathrm{m} / \mathrm{z}$ calculated for $\left[\mathrm{C}_{17} \mathrm{H}_{14} \mathrm{NaO}_{4}\right]^{+}=\left[\mathrm{M}+\mathrm{Na}^{+}\right.$: 305.0784; found: 305.0794 .

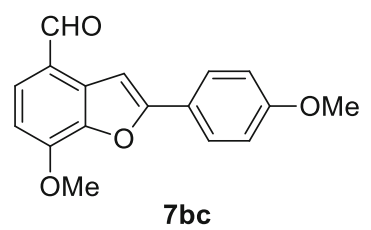

This compound was prepared according to the GP and isolated as brown color solid $91 \%$ yield $(128 \mathrm{mg})$. M.p. $80-82^{\circ} \mathrm{C}$; [TLC (petroleum ether/ethyl acetate $8: 2, \mathrm{R}_{\mathrm{f}}(\mathbf{6} \mathbf{b})=0.40$, $\mathrm{R}_{\mathrm{f}}(\mathbf{4} \mathbf{h})=0.50$, UV detection]. ${ }^{1} \mathrm{HNMR}\left(\mathrm{CDCl}_{3}, 400 \mathrm{MHz}\right)$ : $\delta=10.04(\mathrm{~s}, 1 \mathrm{H}), 7.87(\mathrm{~d}, J=8.8 \mathrm{~Hz}, 1 \mathrm{H}), 7.65(\mathrm{~s}, 1 \mathrm{H})$, $7.63(\mathrm{~d}, J=8.3 \mathrm{~Hz}, 1 \mathrm{H}), 6.97(\mathrm{~d}, J=8.8 \mathrm{~Hz}, 1 \mathrm{H}), 6.85$ $(\mathrm{d}, J=8.3 \mathrm{~Hz}, 1 \mathrm{H}), 4.12(\mathrm{~s}, 3 \mathrm{H}), 3.86(\mathrm{~s}, 3 \mathrm{H}) \mathrm{ppm} .{ }^{13} \mathrm{C}$ $\operatorname{NMR}\left(\mathrm{CDCl}_{3}, 100 \mathrm{MHz}\right): \delta=190.8\left(\mathrm{C}_{\mathrm{q}}\right), 160.6\left(\mathrm{C}_{\mathrm{q}}\right), 159.3$ $\left(\mathrm{C}_{\mathrm{q}}\right), 149.6\left(\mathrm{C}_{\mathrm{q}}\right), 143.8\left(\mathrm{C}_{\mathrm{q}}\right), 131.8(\mathrm{CH}), 130.4\left(\mathrm{C}_{\mathrm{q}}\right), 127.0$ $(\mathrm{CH}), 122.7\left(\mathrm{C}_{\mathrm{q}}\right), 122.5\left(\mathrm{C}_{\mathrm{q}}\right), 114.3(\mathrm{CH}), 105.8(\mathrm{CH}), 100.4$ $(\mathrm{CH}), 56.4\left(\mathrm{CH}_{3}\right), 55.4\left(\mathrm{CH}_{3}\right)$ ppm.IR (MIR-ATR, 4000$\left.600 \mathrm{~cm}^{-1}\right): v_{\max }=2921,2850,1563,1480,1465,1381$, 1121, 1103, 1012, $928 \mathrm{~cm}^{-1}$. HR-MS (ESI $\left.{ }^{+}\right) \mathrm{m} / \mathrm{z}$ calculated for $\left[\mathrm{C}_{17} \mathrm{H}_{14} \mathrm{NaO}_{4}\right]^{+}=[\mathrm{M}+\mathrm{Na}]^{+}$: 305.0784 ; found: 305.0788 .

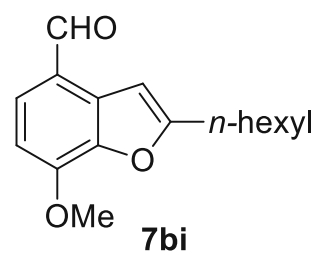

This compound was prepared according to the GP and isolated as brown color viscous liquid $89 \%$ yield $(116 \mathrm{mg})$. [TLC (petroleum ether/ethyl acetate $8: 2, \mathrm{R}_{\mathrm{f}}(\mathbf{6 b})=0.50$, $\mathrm{R}_{\mathrm{f}}(\mathbf{4 i})=0.60$, UV detection]. ${ }^{1} \mathrm{H} \mathrm{NMR}\left(\mathrm{CDCl}_{3}, 400 \mathrm{MHz}\right)$ : $\delta=10.01(\mathrm{~s}, 1 \mathrm{H}), 7.62(\mathrm{~d}, J=8.3 \mathrm{~Hz}, 1 \mathrm{H}), 7.14(\mathrm{~s}, 1 \mathrm{H}), 6.82$ $(\mathrm{d}, J=8.3 \mathrm{~Hz}, 1 \mathrm{H}), 4.08(\mathrm{~s}, 3 \mathrm{H}), 2.81(\mathrm{t}, J=7.3 \mathrm{~Hz}, 2 \mathrm{H})$, 1.85-1.70 (m, 2H), 1.45-1.15 (m, 6H), $0.87(\mathrm{t}, J=7.3 \mathrm{~Hz}$, $3 \mathrm{H}) \mathrm{ppm} .{ }^{13} \mathrm{C} \mathrm{NMR}\left(\mathrm{CDCl}_{3}, 100 \mathrm{MHz}\right): \delta=190.8\left(\mathrm{C}_{\mathrm{q}}\right)$, $163.4\left(\mathrm{C}_{\mathrm{q}}\right), 149.4\left(\mathrm{C}_{\mathrm{q}}\right), 143.6\left(\mathrm{C}_{\mathrm{q}}\right), 131.3(\mathrm{CH}), 129.8\left(\mathrm{C}_{\mathrm{q}}\right)$, $122.6\left(\mathrm{C}_{\mathrm{q}}\right), 105.0(\mathrm{CH}), 102.6(\mathrm{CH}), 56.3\left(\mathrm{CH}_{3}\right), 31.5\left(\mathrm{CH}_{2}\right)$, $28.9\left(\mathrm{CH}_{2}\right), 28.5\left(\mathrm{CH}_{2}\right), 27.6\left(\mathrm{CH}_{2}\right), 22.5\left(\mathrm{CH}_{2}\right), 14.0\left(\mathrm{CH}_{3}\right)$ ppm. IR (MIR-ATR, 4000-600 $\left.\mathrm{cm}^{-1}\right): v_{\max }=2930,2843$, $1560,1481,1456,1375,1187,1160,1009,929 \mathrm{~cm}^{-1}$. HR$\mathrm{MS}\left(\mathrm{ESI}^{+}\right) \mathrm{m} / \mathrm{z}$ calculated for $\left[\mathrm{C}_{16} \mathrm{H}_{20} \mathrm{NaO}_{3}\right]^{+}=[\mathrm{M}+\mathrm{Na}]^{+}$: 283.1305; found: 283.1308 .<smiles>COc1ccc(C=O)c2cc(P(=O)(O)O)oc12</smiles>

This compound was prepared according to the GP and isolated as brown color viscous liquid $86 \%$ yield $(118 \mathrm{mg})$ : [TLC (petroleum ether/ethyl acetate $9: 1, \mathrm{R}_{\mathrm{f}}(\mathbf{6 b})=0.40$, $\mathrm{R}_{\mathrm{f}}(\mathbf{4 j})=0.70$, UV detection]. ${ }^{1} \mathrm{H} \mathrm{NMR}\left(\mathrm{CDCl}_{3}, 400 \mathrm{MHz}\right)$ : $\delta=10.02(\mathrm{~s}, 1 \mathrm{H}), 7.63(\mathrm{~d}, J=8.3 \mathrm{~Hz}, 1 \mathrm{H}), 7.15(\mathrm{~s}$, $1 \mathrm{H}), 6.84(\mathrm{~d}, J=8.3 \mathrm{~Hz}, 1 \mathrm{H}), 4.09(\mathrm{~s}, 3 \mathrm{H}), 2.83(\mathrm{t}$, $J=7.8 \mathrm{~Hz}, 2 \mathrm{H}), 1.85-1.70(\mathrm{~m}, 2 \mathrm{H}), 1.45-1.15(\mathrm{~m}, 8 \mathrm{H})$, $0.88(\mathrm{t}, J=7.8 \mathrm{~Hz}, 3 \mathrm{H}) \mathrm{ppm} .{ }^{13} \mathrm{C} \mathrm{NMR}\left(\mathrm{CDCl}_{3}, 100 \mathrm{MHz}\right)$ : $\delta=190.8\left(\mathrm{C}_{\mathrm{q}}\right), 163.4\left(\mathrm{C}_{\mathrm{q}}\right), 149.4\left(\mathrm{C}_{\mathrm{q}}\right), 143.7\left(\mathrm{C}_{\mathrm{q}}\right), 131.3$ $(\mathrm{CH}), 129.9\left(\mathrm{C}_{\mathrm{q}}\right), 122.6\left(\mathrm{C}_{\mathrm{q}}\right), 105.0(\mathrm{CH}), 102.6(\mathrm{CH}), 56.3$ $\left(\mathrm{CH}_{3}\right), 31.7\left(\mathrm{CH}_{2}\right), 29.1\left(\mathrm{CH}_{2}\right), 29.0\left(\mathrm{CH}_{2}\right), 28.5\left(\mathrm{CH}_{2}\right), 27.6$ $\left(\mathrm{CH}_{2}\right), 22.6\left(\mathrm{CH}_{2}\right), 14.0\left(\mathrm{CH}_{3}\right)$ ppm. IR (MIR-ATR, 4000$\left.600 \mathrm{~cm}^{-1}\right): v_{\max }=2927,2850,1560,1480,1471,1380$, 1190, 1165, 1010, $926 \mathrm{~cm}^{-1}$. HR-MS (ESI $\left.{ }^{+}\right) \mathrm{m} / \mathrm{z}$ calculated for $\left[\mathrm{C}_{17} \mathrm{H}_{22} \mathrm{NaO}_{3}\right]^{+}=[\mathrm{M}+\mathrm{Na}]^{+}: 297.1461$; found: 297.1465 .<smiles>COc1cc2c(C=O)ccc(OC)c2o1</smiles>

This compound was prepared according to the GP and isolated as brown color viscous liquid $90 \%$ yield (129 mg): [TLC (petroleum ether/ethyl acetate $8: 2, \mathrm{R}_{\mathrm{f}}(\mathbf{6 b})=0.50$, $\mathrm{R}_{\mathrm{f}}(4 \mathrm{k})=0.60$, UV detection]. ${ }^{1} \mathrm{HNMR}\left(\mathrm{CDCl}_{3}, 400 \mathrm{MHz}\right)$ : $\delta=10.01(\mathrm{~s}, 1 \mathrm{H}), 7.62(\mathrm{~d}, J=8.3 \mathrm{~Hz}, 1 \mathrm{H}), 7.14(\mathrm{~s}$, $1 \mathrm{H}), 6.83(\mathrm{~d}, J=8.3 \mathrm{~Hz}, 1 \mathrm{H}), 4.08(\mathrm{~s}, 3 \mathrm{H}), 2.81(\mathrm{t}$, $J=7.3 \mathrm{~Hz}, 2 \mathrm{H}), 1.85-1.70(\mathrm{~m}, 2 \mathrm{H}), 1.45-1.15(\mathrm{~m}, 10 \mathrm{H})$, $0.87(\mathrm{t}, J=7.3 \mathrm{~Hz}, 3 \mathrm{H}) \mathrm{ppm} .{ }^{13} \mathrm{C} \mathrm{NMR}\left(\mathrm{CDCl}_{3}, 100 \mathrm{MHz}\right)$ : $\delta=190.8\left(\mathrm{C}_{\mathrm{q}}\right), 163.4\left(\mathrm{C}_{\mathrm{q}}\right), 149.4\left(\mathrm{C}_{\mathrm{q}}\right), 143.7\left(\mathrm{C}_{\mathrm{q}}\right), 131.3$ $(\mathrm{CH}), 129.9\left(\mathrm{C}_{\mathrm{q}}\right), 122.6\left(\mathrm{C}_{\mathrm{q}}\right), 105.1(\mathrm{CH}), 102.6(\mathrm{CH}), 56.3$ 
$\left(\mathrm{CH}_{3}\right), 31.8\left(\mathrm{CH}_{2}\right), 29.3\left(\mathrm{CH}_{2}\right), 29.2\left(\mathrm{CH}_{2}\right), 29.1\left(\mathrm{CH}_{2}\right), 28.5$ $\left(\mathrm{CH}_{2}\right), 27.6\left(\mathrm{CH}_{2}\right), 22.6\left(\mathrm{CH}_{2}\right), 14.1\left(\mathrm{CH}_{3}\right)$ ppm. IR (MIRATR, 4000-600 $\left.\mathrm{cm}^{-1}\right): v_{\max }=2920,2865,1560,1484$, 1463, 1380, 1132, 1009, $926 \mathrm{~cm}^{-1}$. HR-MS (ESI $\left.{ }^{+}\right) \mathrm{m} / \mathrm{z}$ calculated for $\left[\mathrm{C}_{18} \mathrm{H}_{25} \mathrm{O}_{3}\right]^{+}=[\mathrm{M}+\mathrm{H}]^{+}$: 289.1798; found: 289.1801 .<smiles>COc1cc2c(C=O)ccc(OC)c2o1</smiles>

This compound was prepared according to the GP and isolated as pale yellow color liquid 90\% yield (142 mg). [TLC (petroleum ether/ethyl acetate $8: 2, \mathrm{R}_{\mathrm{f}}(\mathbf{6 b})=0.50$, $\mathrm{R}_{\mathrm{f}}(4 \mathrm{l})=0.60, \mathrm{UV}$ detection]. ${ }^{1} \mathrm{H} \mathrm{NMR}\left(\mathrm{CDCl}_{3}, 400 \mathrm{MHz}\right)$ : $\delta=10.01(\mathrm{~s}, 1 \mathrm{H}), 7.62(\mathrm{~d}, J=7.8 \mathrm{~Hz}, 1 \mathrm{H}), 7.14(\mathrm{~s}, 1 \mathrm{H}), 6.83$ $(\mathrm{d}, J=8.3 \mathrm{~Hz}, 1 \mathrm{H}), 4.08(\mathrm{~s}, 3 \mathrm{H}), 2.82(\mathrm{t}, J=7.3 \mathrm{~Hz}, 2 \mathrm{H})$, 1.85-1.70 (m, 2H), 1.45-1.15 (m, 14H), $0.87(\mathrm{t}, J=7.3 \mathrm{~Hz}$, $3 \mathrm{H}) \mathrm{ppm} .{ }^{13} \mathrm{C} \mathrm{NMR}\left(\mathrm{CDCl}_{3}, 100 \mathrm{MHz}\right): \delta=190.8\left(\mathrm{C}_{\mathrm{q}}\right)$, $163.4\left(\mathrm{C}_{\mathrm{q}}\right), 149.4\left(\mathrm{C}_{\mathrm{q}}\right), 143.7\left(\mathrm{C}_{\mathrm{q}}\right), 131.3(\mathrm{CH}), 129.9\left(\mathrm{C}_{\mathrm{q}}\right)$, $122.6\left(\mathrm{C}_{\mathrm{q}}\right), 105.1(\mathrm{CH}), 102.6(\mathrm{CH}), 56.3\left(\mathrm{CH}_{3}\right), 31.9\left(\mathrm{CH}_{2}\right)$, $29.6\left(\mathrm{CH}_{2}\right), 29.5\left(\mathrm{CH}_{2}\right), 29.3\left(2 \mathrm{C}, \mathrm{CH}_{2}\right), 29.2\left(\mathrm{CH}_{2}\right), 28.5$ $\left(\mathrm{CH}_{2}\right), 27.6\left(\mathrm{CH}_{2}\right), 22.7\left(\mathrm{CH}_{2}\right), 14.1\left(\mathrm{CH}_{3}\right) \mathrm{ppm}$. IR (MIRATR, 4000-600 $\left.\mathrm{cm}^{-1}\right): v_{\max }=2921,2851,1530,1483$, 1460, 1321, 1180, 1163, 1107, 1009, $920 \mathrm{~cm}^{-1}$. HR-MS $\left(\mathrm{ESI}^{+}\right) \mathrm{m} / \mathrm{z}$ calculated for $\left[\mathrm{C}_{20} \mathrm{H}_{28} \mathrm{NaO}_{3}\right]^{+}=[\mathrm{M}+\mathrm{Na}]^{+}$: 339.1931; found: 339.1930.

\section{Results and Discussion}

The first part of the report deals with the optimization of conditions for Suzuki-Miyaura coupling. Recently, we reported that $\mathrm{PdO} / \mathrm{GO}$ shows the best activity for Heck coupling, where it showed the best efficacy, turn over number (TON) and turn over frequency (TOF). ${ }^{18}$ Thus, the reaction was explored between iodobenzene 1a and phenylboronic acid 2a in the presence $\mathrm{PdO} / \mathrm{GO}$ of $\mathrm{K}_{2} \mathrm{CO}_{3}$ base in DMSO as solvent at $120^{\circ} \mathrm{C}$. Interestingly, the reaction was quite successful and furnished the biaryl product 3aa, in excellent yield (Table 1, entry 1). On the other hand, the reaction was found to be inferior with the other solvents, such as DMF, DMA, toluene, 1,4-dioxane, THF and acetonitrile (Table 1, entries 2-7). Also, the reaction gave fair to good yields of the product 3aa with the bases $\mathrm{Na}_{2} \mathrm{CO}_{3}$ and $\mathrm{K}_{3} \mathrm{PO}_{4}$, respectively (Table 1, entries 8 to 9).

Table 1 summarizes the optimization conditions of the present study. With these optimized conditions in hand, to extend the scope and generality of the method, the Suzuki-Miyaura coupling was explored between various iodoarenes 1a-g and arylboronic acids $\mathbf{2 a - c .}$
Table 1. Optimization studies for the formation of biphenyl 3aa. ${ }^{a}$

\begin{tabular}{lccc}
\hline & & \\
Entry & Base & Solvent & Yield 3aa $(\%)^{b}$ \\
\hline 1 & $\mathrm{~K}_{2} \mathrm{CO}_{3}$ & DMSO & 90 \\
2 & $\mathrm{~K}_{2} \mathrm{CO}_{3}$ & DMF & 78 \\
3 & $\mathrm{~K}_{2} \mathrm{CO}_{3}$ & DMA & 55 \\
4 & $\mathrm{~K}_{2} \mathrm{CO}_{3}$ & toluene & 43 \\
5 & $\mathrm{~K}_{2} \mathrm{CO}_{3}$ & $1,4-$ dioxane & 70 \\
6 & $\mathrm{~K}_{2} \mathrm{CO}_{3}$ & THF & 64 \\
7 & $\mathrm{~K}_{2} \mathrm{CO}_{3}$ & acetonitrile & 50 \\
8 & $\mathrm{Na}_{2} \mathrm{CO}_{3}$ & DMSO & 72 \\
9 & $\mathrm{~K}_{3} \mathrm{PO}_{4}$ & DMSO & 65 \\
\hline
\end{tabular}

${ }^{a}$ Reaction Conditions: aryl iodides 1a $(0.25 \mathrm{mmol})$, aryl boronic acid $2 \mathbf{a}(0.5 \mathrm{mmol})$, $\mathrm{PdO} / \mathrm{GO}(5 \mathrm{~mol} \%), \mathrm{K}_{2} \mathrm{CO}_{3}(0.5 \mathrm{mmol})$ and DMSO $(1 \mathrm{~mL})$ at $120{ }^{\circ} \mathrm{C}$. ${ }^{b}$ Isolated yields of product 3aa.

Gratifyingly, the reaction was found to be amenable and afforded the corresponding biaryls 3aa-gc, in very good to excellent yields (Table 2). Interestingly, the reaction was successful with simple iodobenzene 1a and also with different functional $\left(\mathrm{CF}_{3}, \mathrm{~F}, \mathrm{Me}\right.$ and $\left.\mathrm{OMe}\right)$ groups on aromatic ring $\mathbf{1 b}-\mathbf{g}$ (Table 2). Notably, the reaction was also amenable with simple arylboronic acid $\mathbf{2 a}$ as well as electron-rich $\mathbf{2 b}$ and electron deficient $\mathbf{2 c}$ aromatic moieties (Table 2). However, in an analogy to our previous report, ${ }^{18}$ the reaction with bromoarenes did not show progress. This may due to the fact the iodoarenes are relatively more reactive than that of bromo ones.

It is worth noting that the catalyst retains its activity, which is evident with nearly no loss of activity until the fifth cycle (Figure 1). This was done by recovering the catalyst by centrifugation and washing with ethyl acetate and acetone followed by drying in a hot air oven at $60{ }^{\circ} \mathrm{C}$ for $12 \mathrm{~h}$. The recovered $\mathrm{PdO} / \mathrm{GO}$ catalyst was then subjected to the next catalytic cycle. The marginal loss of activity after the fifth cycle $(<3 \%)$ may be due to loss of some amount of the catalyst during the recovery of $\mathrm{PdO} / \mathrm{GO}$. The catalyst was recycled five times without appreciable change in the product 3aa yield under the established conditions at $120{ }^{\circ} \mathrm{C}$. Thus, based on the above results it was confirmed that $\mathrm{PdO} / \mathrm{GO}$ catalyst is stable enough and can be reused.

After successful synthesis of biaryls using SuzukiMiyaura coupling, we attempted Glaser coupling. Symmetrical 1,3-diynes comprise an important class of compounds used for the accomplishment of $\pi$-conjugated 
Table 2. Synthesis of biphenyls 3aa-gc with aryl iodides $\mathbf{1 a - g}$ and arylboronic acids $\mathbf{2 a - c}{ }^{a, b}$

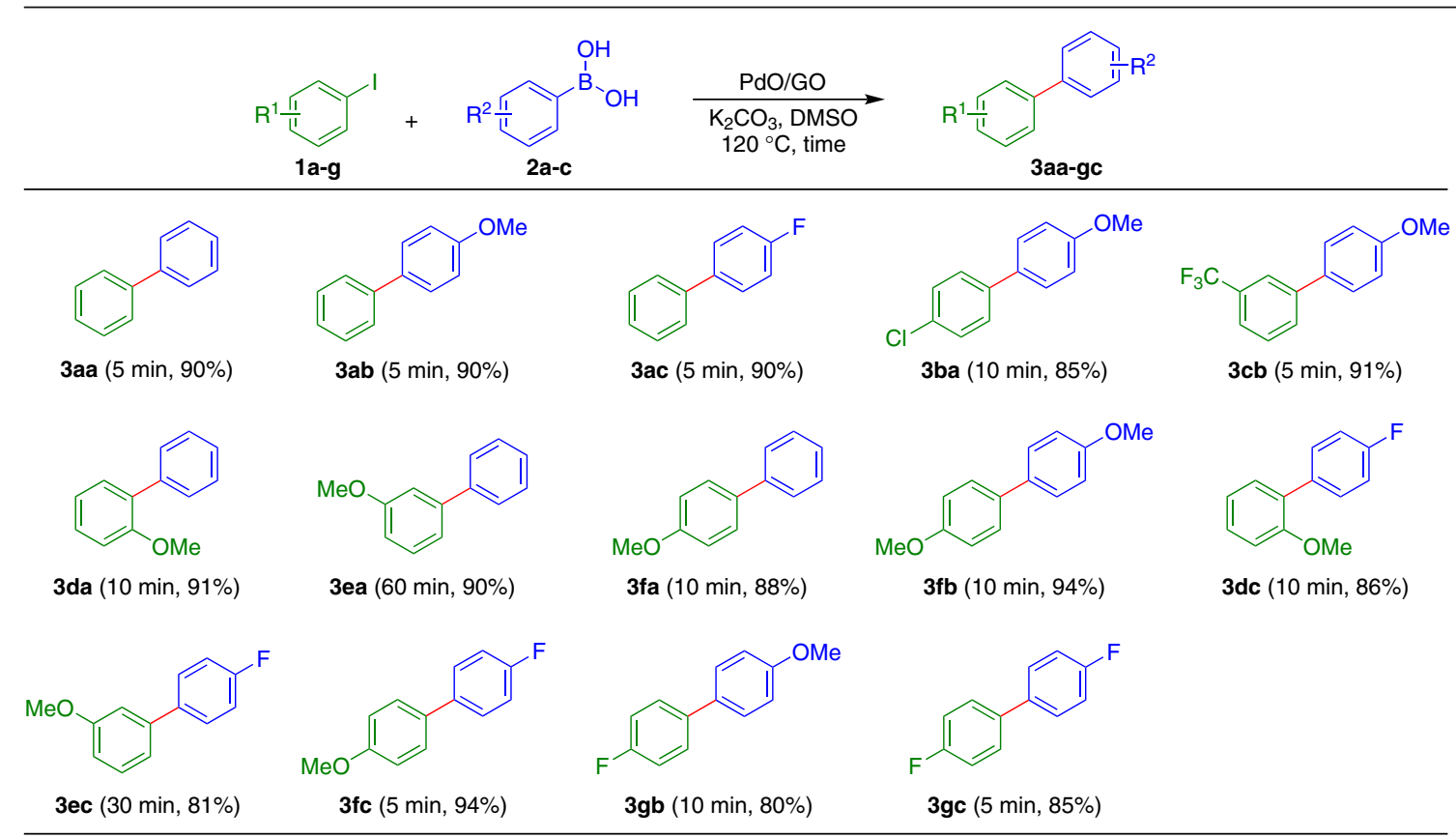

${ }^{a}$ Reaction Conditions: iodobenzene 1a-g $(0.25 \mathrm{mmol})$, arylboronic acid $\mathbf{2 a - c}(0.5 \mathrm{mmol}), \mathrm{PdO} / \mathrm{GO}(5 \mathrm{~mol} \%)$, $\mathrm{K}_{2} \mathrm{CO}_{3}(0.5 \mathrm{mmol})$ and DMSO $(1 \mathrm{~mL})$ at $120{ }^{\circ} \mathrm{C} .{ }^{b}$ Isolated yields of product 3aa-gc.

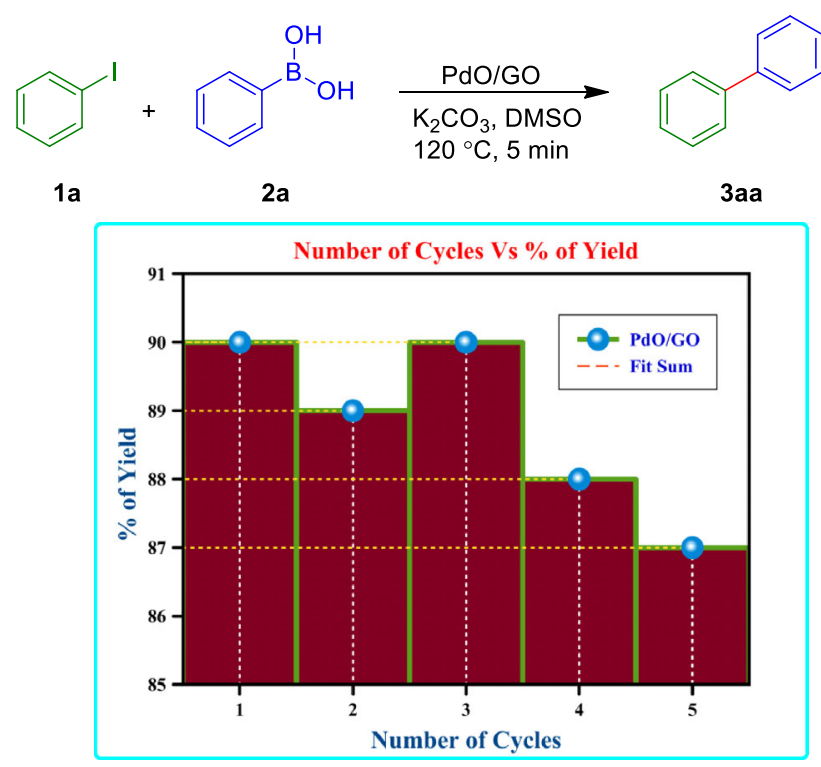

Figure 1. Recyclability of the catalyst in Suzuki reaction.

polymeric materials, supramolecular structures, natural products and pharmaceuticals. ${ }^{20-25}$

The first synthesis of symmetric 1,3-diynes through homo-coupling of terminal alkynes was reported by Carl Glaser in 1869 in the presence of $\mathrm{Cu}(\mathrm{I})$ salt and oxygen in air. ${ }^{26,27}$ Subsequently, Eglinton ${ }^{28}$ and Hay ${ }^{29,30}$ modified the method to improve the versatility of the method. Thereafter, some interesting $[\mathrm{Cu}]$-catalyzed approaches have been developed. Though Glaser coupling was well established under $[\mathrm{Cu}]$-catalysis, there are a few reports by using other transition-metal-catalysts, such as Pd$\mathrm{Cu},{ }^{32,33} \mathrm{Pd}-\mathrm{Ag},{ }^{34} \mathrm{Pd},{ }^{25,35} \mathrm{Ni},{ }^{36} \mathrm{Au},{ }^{37,38}$ etc. Herein, we intended to check the applicability of present $\mathrm{PdO} / \mathrm{GO}$ catalyst for Glaser coupling. Therefore, the reaction was performed with terminal acetylenes $\mathbf{4 a - g}$ under established reactions of Suzuki-Miyaura coupling. As seen from Table 3, the reaction was quite successful and furnished the Glaser coupling products 5a-g, in fair to good yields (Table 3 ).

To further demonstrate the applicability of $\mathrm{PdO} / \mathrm{GO}$ catalyst, one-pot synthesis of benzofurans was tested. Benzofurans are ubiquitous oxygen-containing heterocyclic motifs that constitute many natural products, pharmaceuticals, biologically important compounds and organic materials. In this context, many synthetic strategies have been established for their synthesis. Notably, transition metal-catalyzed $\left(\mathrm{Pd},{ }^{39-47} \mathrm{Rh},{ }^{48} \mathrm{Ru},{ }^{48-52} \mathrm{Ir},{ }^{53}\right.$ $\mathrm{Au}^{54}$ and $\mathrm{Cu}^{55-57}$ ) annulations proved to be powerful strategies for the synthesis of benzofurans. With this background, $\mathrm{PdO} / \mathrm{GO}$ catalyst was employed for the one-pot synthesis of 2-substituted benzofurans starting from 2-iodophenol $\mathbf{6 a}$ and terminal acetylene $\mathbf{4 a}$, under standard conditions. However, the reaction furnished the simple Glaser product through homo-coupling and $\mathrm{PdO} / \mathrm{MWCNT}$ catalyst is the best catalyst and furnished the benzofuran 7aa, in excellent yield (Table 4). 
Table 3. Synthesis of Symmetrical 1,3-diynes5a-g from terminal alkynes4a-g. ${ }^{a, b}$

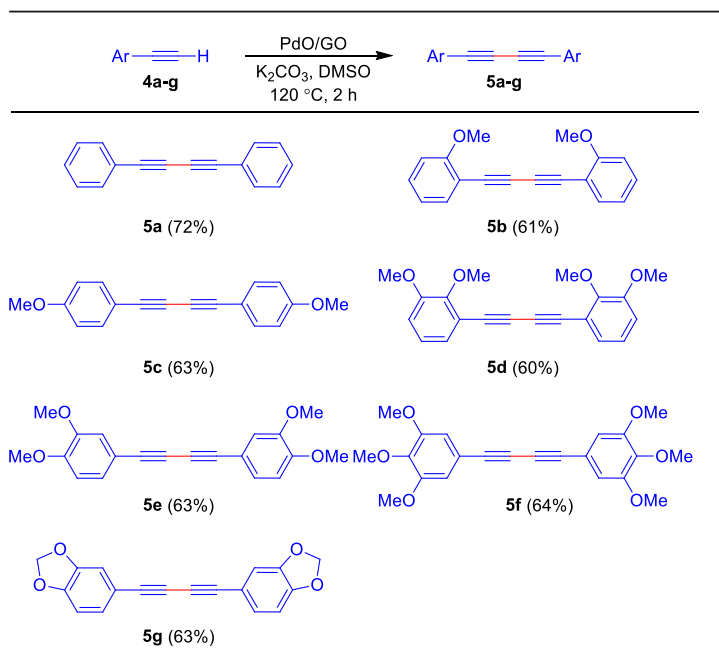

${ }^{a}$ Reaction Conditions: aryl alkynes 4a-g $(0.5 \mathrm{mmol})$, $\mathrm{PdO} / \mathrm{GO}(5 \mathrm{~mol} \%), \mathrm{K}_{2} \mathrm{CO}_{3}(0.10 \mathrm{mmol})$ and DMSO $(1 \mathrm{~mL})$ at $120{ }^{\circ} \mathrm{C} .{ }^{b}$ Isolated yields of product $\mathbf{5 a - g}$.

Table 4. Synthesis of benzofurans 7aa-bl from 2-iodophenols 6a-b and aryl alkynes $\mathbf{4 a - 1} .^{a, b}$

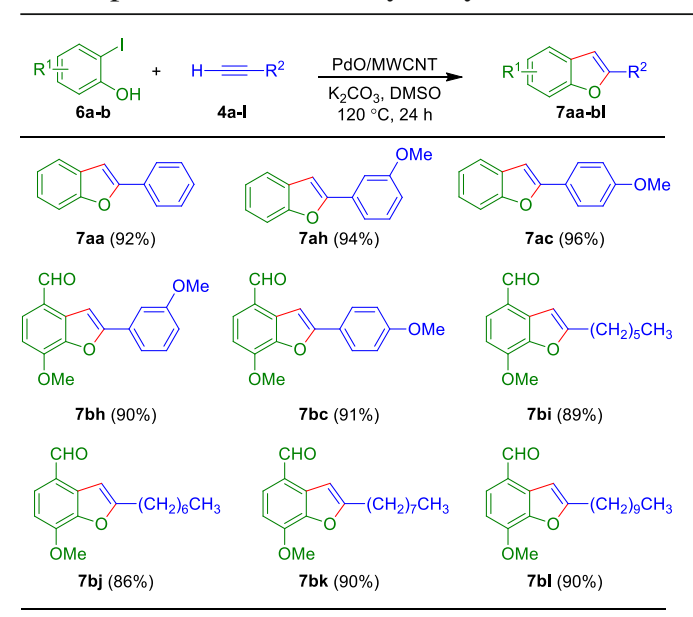

${ }^{a}$ Reaction Conditions: Iodophenol 6a-b (0.5 mmol), arylalkyne 4a-i $(0.5 \mathrm{mmol})$, PdO/MWCNT (5 mol\%), $\mathrm{K}_{2} \mathrm{CO}_{3}$ (1 mmol) and DMSO $(1 \mathrm{~mL})$ at $120{ }^{\circ} \mathrm{C} .{ }^{b}$ Isolated yields of product 7aa-bl.

These conditions were applied to other systems as well. As anticipated, the protocol was quite successful and furnished the 2-substituted benzofurans 7ah-bl, in very good to excellent yields (Table 4). Notably, the reaction was compatible with aryl as well as alkyl terminal acetylenes. Significantly, the reaction was tolerable to the aldehyde functionality on the aromatic ring of iodophenol.

\section{Conclusions}

In summary, an efficient $\mathrm{PdO} / \mathrm{GO}$ nano-catalyst catalyzed Suzuki-Miyaura and Glaser coupling reactions have been demonstrated, for the synthesis of biaryls and 1,3-diynes. Also, domino one-pot synthesis of 2-alkyl/2-aryl benzofurans has been accomplished starting from 2-iodophenols and terminal alkynes, in the presence of $\mathrm{PdO} / \mathrm{MWCNT}$ nano-catalyst. The formation of benzofurans proceeds through intermolecular Sonogashira reaction followed by intramolecular nucleophilic addition of internal hydroxyl group onto the acetylene bond. Catalyst retains its activity even after 5 cycles.

\section{Supplementary Information (SI)}

${ }^{1} \mathrm{H},{ }^{13} \mathrm{C}$ NMR spectras of all isolated products, XRD pattern, SEM images of as-synthesized catalysts are given. Supplementary Information is available at www.ias.ac.in/chemsci.

\section{Acknowledgements}

B.L. thanks the University of Grant Commission (UGC), New Delhi, for the award research fellowship.

\section{References}

1. Lin J, Mei T, Lv M, Zhang C A, Zhao Z and Wang X 2014 Size-controlled PdO/graphene oxides and their reduction products with high catalytic activity RSC Adv. 429563

2. Wang X, Chen W and Yan L 2014 Three-dimensional reduced graphene oxide architecture embedded palladium nanoparticles as highly active catalyst for the SuzukieMiyaura coupling reaction Mater. Chem. Phys. 148103

3. Schaetz A, Zeltner M and Stark W J 2012 Carbon modifications and surfaces for catalytic organic transformations ACS Catal. 21267

4. Nakhate A V and Yadav G D 2016 Palladium nanoparticles supported carbon based graphene oxide monolith as catalyst for sonogashira coupling and hydrogenation of nitrobenzene and alkenes ChemistrySelect. 13954

5. Li H, Johansson Seechurn C C and Colacot T J 2012 Development of preformed Pd catalysts for crosscoupling reactions, beyond the 2010 Nobel Prize ACS Catal. 21147

6. Zhou J, Li X and Sun H J 2010 An efficient and recyclable water-soluble cyclopalladated complex for aqueous Suzuki reactions under aerial conditions Organomet. Chem. 695297

7. Reetz M T and de Vries J G 2004 Ligand-free Heck reactions using low Pd-loading Chem. Commun. 1559

8. Kumbhar A, Kamble S, Mane A, Jha R and Salunkhe R 2013 Modified zeolite immobilized palladium for ligand-free Suzuki-Miyaura cross-coupling reaction $J$. Organomet. Chem. $\mathbf{7 3 8} 29$ 
9. Moussa S, Siamaki A R, Gupton B F and El-Shall M S 2012 Pd-Partially reduced graphene oxide catalysts (Pd/PRGO): Laser synthesis of Pd nanoparticles supported on PRGO nanosheets for carbon-carbon cross coupling reactions ACS Catal. 2145

10. Rao C N R, Sood A K, Subrahmanyam K S and Govindaraj A 2009 Graphene: the new two-dimensional nanomaterial Angew. Chem. Int. Edit. 487752

11. Novoselov K S, Jiang Z, Zhang Y, Morozov S V, Stormer H L, Zeitler U, Maan J C, Boebinger G S, Kim P and Geim A K 2007 Room-temperature quantum hall effect in graphene Science 3151379

12. Novoselov K S, Geim A K, Morozov S V, Jiang D, Katsneloson M L, Grigorieva I V, Dubonos S V and Firsov A A 2005 Two-dimensional gas of massless Dirac fermions in grapheme Nature 438197

13. Duan H, Li M, Zhang G, Gallagher J R, Huang Z, Sun Y and Lei A 2015 Single-site palladium(II) catalyst for oxidative heck reaction: catalytic performance and kinetic investigations ACS Catal. 53752

14. Zhou P X, Ye Y Y, Liu C, Zhao L B, Hou J Y, Chen D Q and Xu P F 2015 Palladium-Catalyzed Acylation/Alkenylation of Aryl Iodide: A Domino Approach Based on the Catellani-Lautens Reaction ACS Catal. 5 4927

15. Larhed M and Hallberg A 2001 Microwave-assisted high-speed chemistry: a new technique in drug discovery Drug Discov. Today 6406

16. Yar M S and Ansari Z H 2009 Synthesis and in vivo diuretic activity of biphenyl benzothiazole2-carboxamide derivatives Acta Pol. Pharm. 66 387

17. Williams D A and Lemke T L 2002 Foye's Principles of Medical Chemistry 15th edn. (Philadelphia: Lippincott Williams \& Wilkins) p. 533

18. Narayana B L, Mahendar L, Ghosal P, Satyanarayana G and Subrahmanyam Ch 2017 Nano-sized recyclable $\mathrm{PdO}$ supported carbon nanostructures for heck reaction: influence of carbon materials ChemistrySelect. 22703

19. Narayana B L, Mukri B D, Ghosal P and Subrahmanyam Ch 2016 Mn Ion substituted $\mathrm{CeO}_{2}$ nano spheres for low temperature $\mathrm{CO}$ oxidation: the promoting effect of $\mathrm{Mn}$ ions ChemistrySelect. 13150

20. Nicolaou K C, Petasis N A and Zipkin R E 1982 The endiandric acid cascade. Electrocyclizations in organic synthesis. 4. Biomimetic approach to endiandric acids A-G. Total synthesis and thermal studies J. Am. Chem. Soc. 1045560

21. Zhou L, Zhan H Y and Jiang H F 2007 An efficient and practical process for $\mathrm{Pd} / \mathrm{Cu}$ cocatalyzedhomocoupling reaction of terminal alkynes using sodium percarbonate as a dual reagent in aqueous media Chin. J. Chem. 25 1413

22. Siemsen P, Livingston R C and Diederich F 2000 Acetylenic coupling: a powerful tool in molecular construction Angew. Chem. Int. Edit. 392632

23. Zhang L Y and Wang L 2008 Immobilized palladium on organic-inorganic hybrid materials: a novel and reusable catalyst for the copper-free sonogashira coupling reaction Chin. J. Chem. 261601

24. Tour J M 1996 Conjugated macromolecules of precise length and constitution. Organic synthesis for the construction of nanoarchitectures Chem. Rev. 96 537

25. Yin L and Liebscher 2007 Carbon-carbon coupling reactions catalyzed by heterogeneous palladium catalysts $J$. Chem. Rev. 107133

26. Glaser C 1869 Beiträge zur Kenntniss des Acetenylbenzols Ber. Dtsch. Chem. Ges. 2422

27. Glaser C 1870 Untersuchungen über einige Derivate der Zimmtsäure Ann. Chem. Pharm. 154137

28. Eglinton $\mathrm{G}$ and Galbraith R 1959 Macrocyclic acetylenic compounds. Part I. Cyclotetradeca-1:3-diyne and related compounds J. Chem. Soc. 0889

29. Hay A S 1960 Communications-oxidative coupling of acetylenes J. Org. Chem. 251275

30. Hay A S 1962 Oxidative coupling of acetylenes J. Org. Chem. 273320

31. Merkul E, Urselmann D and Muller T 2011 Consecutive one-pot Sonogashira-Glaser coupling sequence - direct preparation of symmetrical diynes by sequential $\mathrm{Pd} / \mathrm{Cu}$ catalysis Eur. J. Org. Chem. 2238

32. Lei A, Srivastava M and Zhang X 2002 Transmetalation of palladium enolate and its application in palladium-catalyzed homocoupling of alkynes: a roomtemperature, highly efficient route to make diynes J. Org. Chem. 671969

33. Li J H, Liang Y and Xie Y X 2005 Efficient palladiumcatalyzed homocoupling reaction and Sonogashira crosscoupling reaction of terminal alkynes under aerobic conditions J. Org. Chem. 704393

34. Guo M, Chen B, Lv M, Zhou X, Wen Y and Shen X 2016 The homocoupling reaction of aromatic terminal alkynes by a highly active palladium(II)/AgNO3 cocatalyst in aqueous media under aerobic conditions Molecules 21 606

35. Rossi R, Carpita A and Bigelli C 1985 A palladiumpromoted route to 3-alkyl-4-(1-alkynyl)-hexa-1,5-dyn3-enes and/or 1,3-diynes Tetrahedron Lett. 26523

36. Yin W, He C, Chen M, Zhang H and Lei A 2009 Nickelcatalyzed oxidative coupling reactions of two different terminal alkynes using $\mathrm{O}_{2}$ as the oxidant at room temperature: facile syntheses of unsymmetric 1,3-diynes $\mathrm{Org}$. Lett. 11709

37. Leyva-Perez A, Domenech A, Al-Resayes S I and Corma A 2012 Gold redox catalytic cycles for the oxidative coupling of alkynes ACS Catal. 2121

38. Zhu M, Ning $\mathrm{M}, \mathrm{Fu} \mathrm{J} \mathrm{W,} \mathrm{Xu} \mathrm{C} \mathrm{and} \mathrm{Zou} \mathrm{G} \mathrm{L}$ 2012 Gold-catalyzed homocoupling reaction of terminal alkynes to 1,3-diynes Bull. Korean Chem. Soc. 33 1325

39. Kuram M R, Bhanuchandra M and Sahoo A K 2013 Direct access to benzo[b]furans through palladiumcatalyzed oxidative annulation of phenols and unactivated internal alkynes Angew. Chem. Int. Edit. 52 4607

40. Castro C E, Gaughan E J and Owsley D C 1966 Indoles, benzofurans, phthalides, and tolanes via copper(I) acetylides J. Org. Chem. 314071

41. Arcadi A, Marinelli F and Cacchi S 1986 Palladiumcatalyzed reaction of 2-hydroxyaryl and hydroxyheteroaryl halides with 1-alkynes: an improved route to the benzo [b] furan ring system Synthesis 9 749 
42. Larock R C, Yum E K, Doty M J and Sham K K C 1995 Synthesis of aromatic heterocycles via palladiumcatalyzed annulation of internal alkynes J. Org. Chem. 60 3270

43. Sanz R, Castroviejo M P, Fernandez Y and Fananas F J 2005 A new and efficient synthesis of 4-functionalized benzo[b]furans from 2,3-dihalophenols J. Org. Chem. 70 6548

44. Gill G S, Grobelny D W, Chaplin J H and Flynn B L 2008 An efficient synthesis and substitution of 3-aroyl2-bromobenzo[b]furans J. Org. Chem. 731131

45. Akai N, Uchida N and Konakahara T 2008 Facile and efficient synthesis of polyfunctionalized benzofurans: three-component coupling reactions from an alkynylsilane, an o-hydroxybenzaldehyde derivative, and a secondary amine by a $\mathrm{Cu}(\mathrm{I})-\mathrm{Cu}(\mathrm{II})$ cooperative catalytic system Tetrahedron Lett. 493437

46. Wang J R and Manabe K 2010 Hydroxyterphenylphoshine-palladium catalyst for benzo[b]furan synthesis from 2-chlorophenols. Bifunctional ligand strategy for cross-coupling of chloroarenes. J. Org. Chem. 755340

47. Wang R, Mo S, Lu Y and Shen Z 2011 Domino Sonogashira coupling/cyclization reaction catalyzed by copper and ppb levels of palladium: a concise route to indoles and benzo[b]furans Adv. Synth. Catal. 353713

48. Zhou Z, Liu G, Shen Y and Lu X 2014 Synthesis of benzofurans via ruthenium-catalyzed redox-neutral $\mathrm{C}-\mathrm{H}$ functionalization and reaction with alkynes under mild conditions Org. Chem. Front. 11161

49. Yeh C H, Chen W C, Gandeepan P, Hong Y C, Shih C $\mathrm{H}$ and Cheng C H 2014 Rh(III)-catalyzed dual directing group assisted sterically hindered $\mathrm{C}-\mathrm{H}$ bond activation: a unique route to meta and ortho substituted benzofurans Org. Biomol. Chem. 129105

50. Lee D H, Kwon K H and Yi C S, 2012 Dehydrative C$\mathrm{H}$ alkylation and alkenylation of phenols with alcohols: expedient synthesis for substituted phenols and benzofurans J. Am. Chem. Soc. 1347325

51. Thirunavukkarasu V S, Donati M and Ackermann L 2012 Hydroxyl-directed ruthenium-catalyzed C-H bond functionalization: versatile access to fluorescent pyrans $\mathrm{Org}$. Lett. 143416

52. Mochida S, Shimizu M, Hirano K, Satoh T and Miura M 2010 Synthesis of naphtho[1,8-bc]pyran derivatives and related compounds through hydroxy group directed $\mathrm{C}-\mathrm{H}$ bond cleavage under rhodium catalysis Chem. Asian J. 5 847

53. Tsuchikama K, Hashimoto Y K, Endo K and Shibata T 2009 Iridium-catalyzed selective synthesis of 4-substituted benzofurans and indoles via directed cyclodehydration Adv. Synth. Catal. 3512850

54. Liao J, Guo P and Chen Q 2016 Au-catalyzed synthesis of benzofurans from phenols and alkynes using molecular oxygen Catal. Commun. 7722

55. Bonnamour J, Piedrafita M and Bolm C 2010 Iron and copper salts in the synthesis of benzo[b]furans $A d v$. Synth. Catal. 3521577

56. Zhu R, Wei J and Shi Z 2013 Benzofuran synthesis via copper-mediated oxidative annulation of phenols and unactivated internal alkynes Chem. Sci. 4 3706

57. Zeng W, Wu W, Jiang H, Huang L, Sun Y, Chen Z and Li X 2013 Facile synthesis of benzofurans via coppercatalyzed aerobic oxidative cyclization of phenols and alkynes Chem. Commun. 496611 